\title{
Investigation of the Thermal Contact Resistance across Aluminium Interface
}

\author{
Chavan N.L*and Sarje S.H \\ JSPM's Imperial College of Engineering \& Research, Wagholi, Pune-14, India \\ Accepted 15 June 2016, Available online 20 June 2016, Special Issue-5 (June 2016)
}

\begin{abstract}
An experimental setup to measure the thermal contact conductance across aluminium interface is described, and the results obtained are presented. The resulting thermal contact resistance data are used in various application of heat transfer. Several factors influence the heat transfer across solid interface. These include the material properties, interface pressure, flatness and roughness of the contacting surfaces, temperature, and interstitial material if any. Results presented show the variation of thermal contact conductance as a function applied interface pressure for aluminium. Various interstitial materials investigated include indium foil, silver foil, and liquid eutectic, as expected, thermal contact resistance decreases as interface pressure increases, except in case of the eutectic, in which it was nearly constant. The softer the interstitial material, the lower the thermal contact resistance. Liquid metal provides the lowest thermal contact resistance across the aluminium interface, followed by the indium foil, and then silver foil.
\end{abstract}

Keywords: Thermal contact resistance, aluminium interface, heat transfer

\section{Introduction}

The issue of Thermal Contact Resistance across metallic interface has been investigated for many situations over the past several decades. When heat flows through two bodies placed in contact, there is a microscopic temperature discontinuity at the interface this temperature discontinuity result from thermal contact resistance.Thermal contact conductance at the metal interface can be improved by increasing actual contact area. This can be done by one or more of the following: (1) making aluminium contact surface very smooth and flat. (2) Applying high pressure at interface. (3) Using soft interstitial material (soft metal such as Indium, liquid or variety of conductive greases).Another problem is a potentially great influence of finite Thermal Contact Resistances (TCR) between the sample and other element of the measurement system. This problem is particularly significant if air is present on the contact surfaces are rough and filled with air. When random rough surfaces are placed in mechanical contact, real contact occurs at the summit of surface asperities which are called microcontacts.To study constriction/spreading resistance of microcontacts, the joint is usually studied in a vacuum where the heat transfer between contacting bodies occur only via conduction through microcontacts.thermal contact resistance (TCR) of

*Corresponding author: Chavan N.L conforming rough surfaces in a vacuum is proportional to the real contact area.

\section{Literature Survey}

Nenad stepanac, Nenad Milosevi studied on guarded hot plate method which is a standard method for the measurements of thermal conductivity of solids in the range from 0.1 to $10 \mathrm{~W} / \mathrm{mK}$. It is used for materials such as ceramics, polymers, materials of biological origin, and other. The method can be applied in a wide working temperature range, from $80 \mathrm{~K}$ up to $1200 \mathrm{~K}$. The main principle of the method is one-dimensional steady-state heat conduction through a sample under test. This method is a potentially great influence of finite thermal contact resistances between the sample and other elements of the measurement system on measurement results. This problem is particularly significant if air is present on the contact surfaces are rough and filled with air. In this case, the effect of the finite contact resistances on the final measurement result can be significantly reduced by applying external mechanical pressure or by inserting a thin layer of some fluid or solid with high thermal conductivity.

MajidBahrami, M. Michael Yovanovich, J. Richard Culham studied on random rough surfaces are when placed in mechanical contact, real contact occurs at the summit of surface asperities which are called microcontacts. The real contact area, $\mathrm{Ar}$, the summation of the microcontacts, forms a small portion 
of the nominal contact area, typically a few percent of the nominal contact area. To study the constriction/spreading resistance of microcontacts, the joint is usually studied in a vacuum where the heat transfer between contacting bodies occursonly via conduction through microcontacts. Thermal contact resistance (TCR) of conforming rough surfaces in a vacuum is proportional to the real contact area . TCR can be decreased by reducing the roughness and outof-flatness of the surfaces before the interface is formed or by increasing the contact pressure. However, manufacturing highly finished surfaces is not practical due to cost constraints. Very little has been done for light pressures $<0.1 \mathrm{MPa}$, which is the applicable range for microelectronics devices. Existing models such ascan accurately predict TCR for moderate to high contact pressures. Milanez et al.experimentally studied low contact pressure joints in a vacuum and showed that the modelsoverestimate the TCR at low pressures. They called this phenomenon the truncation effect and attributed this trend to the Gaussian assumption of the surface asperities which implies that asperities with "infinite" heights exist. Milanezetal.proposed correlations for maximum asperities heights as functionsof surface roughness

A.M. Khounsary, D. Chojnowski, and L. Assoufid focused on the parameter which considered Thermal resistance in practical terms (or its inverse, the conductance depends on (1) material hardness, (2) roughness and flatness of the surfaces, (3) interstitial material, if any, (4) applied interface force, (5) thermal conditions, and (6) other less significant factors Thermal contact conductance at the Si-Cu interface can be improved by increasing the actual contact area. This can be done by one or more of the following: (1) making copper and silicon contact surfaces very smooth and flat, (2) applying a high pressure at the interface, and (3) using a soft interstitial material (soft metals such as indium, liquid metals, or a variety of conductive greases or liquids). When heat flows through two bodies placed in contact, there is a macroscopic temperature discontinuity at the interface. This temperature discontinuity results from thermal contact resistance. For two surfaces in contact, even those that are visually very smooth and flat, the actual contact area is typically less than $10 \%$ of the total area. It is at these areas that the heat flows from one body to the other. Because of heat transfer paths, the heat flux is therefore not uniform in the bodies near the interface. At some distance further away from the interface, the heat flux distributes throughout the materials and can be considered as one-dimensional heat flow. Because of the nonhomogeneous temperature distribution in a cross-sectional area, cylinder temperature measurements should not be taken close to the interface. It is due to this behavior that the thermocouples in the experiment are not positioned closer than $5 \mathrm{~mm}$ from the interface.

G.V Krishna reddy, designed a novel method to reduce the thermal contact resistance at the interface between the heat sink and the computer processor. Three different types of models are verified in this paper. Grease G Is high conducting grease or low conducting grease. The advantage of low conducting grease is that it does not short the internal circuity of the processor at the interface and hence the life of the processor is high. The other two models have been devised to improve the conductance while protecting the processor with a low conducting grease. It is proved experimentally that by adding additional highly conducting layers the thermal resistance drops.

Mirmira, S.R., Marrotta, E.E. and Fletcher, L.S focused on adhesives which are used as filler materials or interstitial material such as ceramics or metal are widely used by the electronics industries in a variety of application, including the attachment of heat sink or spreaders, die attach,underfill and encapsulation. These adhesives typically include large concentrations of filler materials intended to enhance the relatively poor thermal conductivity of the base material. A method is presented for the measurement of these thermal properties using a steady-state guarded heat flux meter test apparatus based on the well documented and familiar ASTM test standard D5470.Five different adhesive materials are tested and a linear fit of the resulting resistance verses thickness data are used to determine the bulk thermal conductivity and contact resistance values. Four of the five materials tested had conductivity values of less than $1 \mathrm{~W} / \mathrm{mK}$. In this paper he suggests that the thermal conductivity of the bulk materials is a constant value that is not affected by the thickness of adhesive layer.

T.McWaid, T.E.Marschall discussed the method of determining thermal contact resistance of wavy surface. For determining the thermal contact resistance of real contacts, a new method has been presented. In this method, assuming a regular sinusoidal surface profile, the real contact area in terms of applied forces has been determined. And thermal contact resistance has been calculated. In this ,the experimental and analysis results have been compared. The result of experiments and analysis had good agreement with each other.

ju lio focused on the solid materials which is used in electronic industries. Also measure the thermal contact resistance across solid materials.

\section{Problem Statement}

The issue of Thermal Contact Resistance across metallic interface has been investigated for many situations over the past several decades. When heat flows through two bodies placed in contact, there is a microscopic temperature discontinuity at the interface ,this temperature discontinuity result from thermal contact resistance.

Another problem is a potentially great influence of finite Thermal Contact Resistances (TCR) between the sample and other element of the measurement system. This problem is particularly significant if air is present on the contact surfaces are rough and filled with air. When random rough surfaces are placed in mechanical 
contact, real contact occurs at the summit of surface asperities which are called microcontacts

To study constriction/spreading resistance of microcontacts, the joint is usually studied in a vacuum where the heat transfer between contacting bodies occur only via conduction through microcontacts.thermal contact resistance(TCR) of conforming rough surfaces in a vacuum is proportional to the real contact area. From above literature Review the following objectives are defined,

1)Prime objectives of the proposed investigations are study the concept of the thermal contact resistance(TCR) and parameter involved in the measurement such as temperature across the test specimen, wattage provided to the heating side of test specimen, heat carried away by cooling side of test specimen.

2)Study of the factor affecting on the TCR by keeping the other parameter constant which involved the relation between TCR and surface roughness of specimen. Study of the relation of the factor affecting on TCR \& heat transfer rate by the graphical method involves graph of heat transfer rate and TCR for the different test specimen having different surface roughness. This method also study the same concept by using different thermal interface materials between test specimen.

3) One of important objectives of this methodology for the experimental set up is to find out remedies to reduce thermal contact resistance so as we can increase heat transfer rate effectively for the industrial applications.

\section{Methodology}

\section{1 specification of experimental setup}

Plate $1: \emptyset 150 \times 8 \mathrm{~mm}$ thick

Plate $2: \emptyset 150 \times 8 \mathrm{~mm}$ thick

Heater : 200 watt $(\varnothing 100 \times 2 \mathrm{~mm})$

Ammeter : 1-5 amp AC

Voltmeter : 0 -500 volt AC

Heating plate(copper plate) : $\emptyset 150 \times 20 \mathrm{~mm}$ thick

Cooling plate(aluminium plate) : $\emptyset 150 \times 20 \mathrm{~mm}$ thick

Thermocouple(K type) : bulb $(0-400){ }^{0} \mathrm{C}$

Temperature indicator : 4 channel $(0-400)^{0} \mathrm{C}, \mathrm{K}$ type thermocouple input of $0-20 \mathrm{~mA}$

\subsection{Experimental procedure}

Set up consist the arrangement of the specimen between the heating and cooling side so there are the constant heat flow from the heating to cooling side. The two test specimen are mounted on each other and by arranging the bolting arrangement, pressure is applied on it so the contact between the plates should be air tight. On the heating plate transferring the heat to the test specimen is of the copper. The heater made of the MICA is fixed on the one side and wattage provided to the heater is measured by the voltmeter and ammeter and control of the heat energy provided is by using the dimmer.

On the other side of set up there is cooling arrangement foe the rejection of the heat flowing through the test specimen. These cooling arrangement plates are made of aluminium for better cooling. For the fast cooling process water cooling arrangement is done in the plate with the water inlet and outlet arrangement. This whole setup is mounted on the table which is madeof the CI square pipe so it give the rigid support to the experiment.

On the one side of the this stand support vertical control panel is provided so the parameter required to measure can be seen and also parameter wants to be controlled can be controlled by the switches and meter also mounted on the control panel. This control panel isconsist of temperature indicator for the indication of the temperature across the plate.ammeter and voltmeter isprovided on the panel so it indicate the energy provided to the heater. Dimmer is also mounted on the panel which will controls the current provided to the heater.following fig 2 shows microscopic view of contact between metal surface.

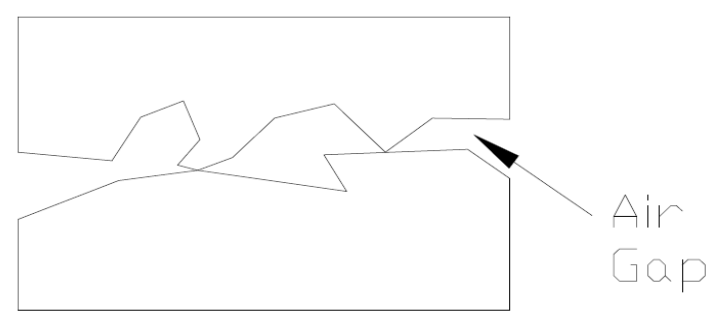

Fig.2-Microscopic view of surface contact

The following fig.3 shows general set up of thermal contact resistance across aluminium interface in which heating arrangement on one side and cooling arrangement on other side. Heat flows from heating to cooling side through the aluminium test specimen.

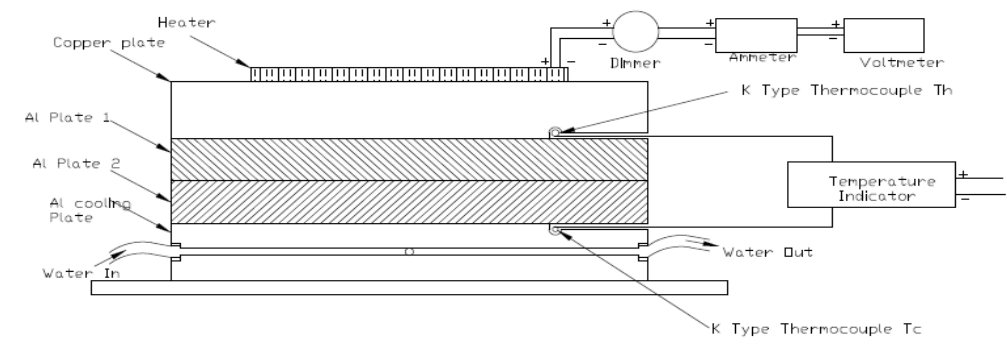

Fig. 3-General experimental setup of thermal contact resistance across aluminium surface 


\section{Observation Table}

Surface roughness is measured by using the surface roughness measuring instrument, these value are for $25 \mathrm{~mm}$ length and this instrument crate thevalues depending the average of deep and cruest present in the $25 \mathrm{~mm}$ measured surface The different temperature reading taken from different test of setup as following tables

Table 1 Surface finish between al plate $\mathrm{Ra}=0.625$

\begin{tabular}{|c|c|c|c|}
\hline Sr No & $\begin{array}{c}\text { Temperature at } \\
\text { Hot side Th }\end{array}$ & $\begin{array}{c}\text { Temperature at } \\
\text { Cold side Tc }\end{array}$ & I X V \\
\hline 1 & 45 & 32 & $53.12 \mathrm{~W}$ \\
\hline 2 & 48 & 28 & $82.4 \mathrm{~W}$ \\
\hline
\end{tabular}

Table 2 Surface finish between Al Plate $\mathrm{Ra}=0.125$

\begin{tabular}{|c|c|c|c|}
\hline Sr No & $\begin{array}{c}\text { Temperature at } \\
\text { Hot side Th }\end{array}$ & $\begin{array}{c}\text { Temperature at } \\
\text { Cold side Tc }\end{array}$ & I X V \\
\hline 1 & 46 & 28 & $54.23 \mathrm{~W}$ \\
\hline 2 & 48 & 37 & $83.62 \mathrm{~W}$ \\
\hline
\end{tabular}

Table 3 Surface contact using fluidic media

\begin{tabular}{|c|c|c|c|}
\hline Sr No & $\begin{array}{c}\text { Temperature at } \\
\text { Hot side }\end{array}$ & $\begin{array}{c}\text { Temperature at } \\
\text { Cold side }\end{array}$ & I X V \\
\hline 1 & 46 & 24 & $52.23 \mathrm{~W}$ \\
\hline 2 & 55 & 22 & $85.16 \mathrm{~W}$ \\
\hline
\end{tabular}

Calculation Part

Surface Area $=\left(\pi r^{2}\right) / 4=3.14 \times 0.15^{2}=0.07065$

1heat transfer from cold to hot section

$\mathrm{Q}=\mathrm{kA}(\mathrm{Th}-\mathrm{Tc}) / \mathrm{L}$

\section{HeatGenrated}

Qgen=Aamp x V volts

3. Heat Transfer Rate at $\mathrm{Ra}=0.625$

$=0.237 \mathrm{x} 0.01767(45-32) / 0.0015=36.29 \mathrm{w} / \mathrm{m} 2$

4.Heat Transfer rate at $\mathrm{Ra}=0.125$

$=0.237 \mathrm{x} 0.01767(46-28) / 0.0015=50.28 \mathrm{w} / \mathrm{m} 2$

5 Heat Transfer when contact using fluidic media

$=0.237 \mathrm{x} 0.01767(46-22) / 0.0015=67 \mathrm{w} / \mathrm{m} 2$

\section{Result and Discussion}

In this session thermal contact resistance is discussed and analyzed effective parameters such as Surface roughness is studied.

\subsection{Effect of surface Roughness}

At Ra value 0.625 heat transfer rate is $36.29 \mathrm{w} / \mathrm{m} 2$ and at value 0.00 ra value heat transfer rate is $67 \mathrm{w} / \mathrm{m} 2$ Contacting face between two similar or dissimilar material may largely affect the rate of heat transfer as the high roughness provide more space in between the plate, so its directly found that high roughness gives low rate of heat transfer. Heat Transfer and roughness of surface are directly affected each other and relation between them involves the term thermal contact resistance.

\subsection{Effect on Heat loss}

As the surface roughness increases the heat loss that is consider here is difference between the heat generated and heat transfer rate is also reduced. Contacting surface of any two similar dissimilar material plates are very largely affect the heat loss quantity low contact resistance may lead to minimize heat loss

\subsection{Remidies for Maximum Heat Transfer and Minimum heat loss}

As third reading provide in Result table show that putting the fluidic i.e. heat transfer rate through involving the fluid is maximum media reduce the surface roughness and hence increase transfer rate Providing the good surface or inserting additives like fluidic media can minimize the resistance for heat flow whole study shows that in any energy heat equipment's involved in surface contact heat transfer are affect majorly due thermal contact resistance that are difficult to calculated.

Below results directly shows us how the term involved in loss of heat during transfer through to different body

Table 4 Experimental parameter reading

\begin{tabular}{|c|c|c|c|c|}
\hline Sr No & Q gen & Ra value & Heat Transfer Rate & T $_{\text {Ho }}$ \\
\hline 1 & 82.4 & 0.625 & 36.29 & 45 \\
\hline 2 & 83.6 & 0.125 & 50.28 & 46 \\
\hline 3 & 86.15 & 0.00 & 67.00 & 46 \\
\hline
\end{tabular}

\section{Conclusions}

In this project work, study found that thermal contact resistance between two surface are measurable and also can be reduced in heat transferring media this method can be utilized to increase the effectiveness of the set up or plant heat generation and heat gains at the end can be maximize through involving the term Thermal contact resistance that may force you to study the surface roughness improving technique

\section{References}

Nenad stepanac,nenadMilosevic,(2009), Correction on the Influence of Therma Contact Resistance in Thermal Conductivity Measurements Using the Guarded Hot Plate Method, Serbian journal of electrical engineering, vol.6, No.3, pp. 479488.

MajidBahrami, M. Michael Yovanovich, J. Richard Culham,(2005), Thermal contact resistance at low contact pressure:Effect of elastic deformation, internationa journal of heat and mass transfer, vol.48, pp. 3284-3293

A.M. Khounsary, D. Chojnowski, and L. Assoufid,(2004), Thermal contact resistance across a copper-silicon interface,SPIE vol.31

G.V Krishna Reddy, N.Chikkanna, B.Uma Maheswar Gowd,(2012), A Novel method to reduce the thermal contact resistance, International journal of recent technology and engineering, vol.1,Issu-2, ISSN: 2277-38

Mirmira, S.R., Marrotta, E.E. and Fletcher, L.S,(1997), Thermal contact conductance of adhesives for microelectronics systems,AIAA Journal of Thermophysics and hea transfer, Vol. 11,No. 2,pp.141-145

T.McWaid, T.E.Marschall,(1992), Thermal contact resistance across pressed metal contacts in vaccum environment, International.Journal of Heat Mass Transfer vol. $35(1$

ju lio(2010), A Simple setup to test thermal contact resistance between interfaces of two contacted solid materials,Electronics packaging technology,PP.116-120 